\title{
Is all motivation good for learning? Dissociable influences of approach and avoidance motivation in declarative memory
}

\author{
Vishnu P. Murty, ${ }^{1,2}$ Kevin S. LaBar, ${ }^{1,3}$ Derek A. Hamilton, ${ }^{4}$ and R. Alison Adcock ${ }^{1,5,6}$ \\ ${ }^{1}$ Center for Cognitive Neuroscience, Duke University, Durham, North Carolina 27708, USA; ${ }^{2}$ Department of Neurobiology, Duke \\ University, Durham, North Carolina 27708, USA; ${ }^{3}$ Psychology and Neuroscience, Duke University, Durham, North Carolina 27708, \\ USA; ${ }^{4}$ Department of Psychology, University of New Mexico, Albuquerque, New Mexico 87131, USA; ${ }^{5}$ Department of Psychiatry, \\ Duke University, Durham, North Carolina 27708, USA
}

\begin{abstract}
The present study investigated the effects of approach versus avoidance motivation on declarative learning. Human participants navigated a virtual reality version of the Morris water task, a classic spatial memory paradigm, adapted to permit the experimental manipulation of motivation during learning. During this task, participants were instructed to navigate to correct platforms while avoiding incorrect platforms. To manipulate motivational states participants were either rewarded for navigating to correct locations (approach) or punished for navigating to incorrect platforms (avoidance). Participants' skin conductance levels (SCLs) were recorded during navigation to investigate the role of physiological arousal in motivated learning. Behavioral results revealed that, overall, approach motivation enhanced and avoidance motivation impaired memory performance compared to nonmotivated spatial learning. This advantage was evident across several performance indices, including accuracy, learning rate, path length, and proximity to platform locations during probe trials. SCL analysis revealed three key findings. First, within subjects, arousal interacted with approach motivation, such that high arousal on a given trial was associated with performance deficits. In addition, across subjects, high arousal negated or reversed the benefits of approach motivation. Finally, low-performing, highly aroused participants showed SCL responses similar to those of avoidance-motivation participants, suggesting that for these individuals, opportunities for reward may evoke states of learning similar to those typically evoked by threats of punishment. These results provide a novel characterization of how approach and avoidance motivation influence declarative memory and indicate a critical and selective role for arousal in determining how reinforcement influences goal-oriented learning.
\end{abstract}

[Supplemental material is available for this article.]

Memories are not direct reflections of the environment, but are instead selective. How does this selectivity arise? Prior research suggests that motivation enhances learning and memory for behaviorally relevant information (Shohamy and Adcock 2010), but there are qualitatively different ways of motivating individuals to learn. For example, a student could equally be motivated to perform well on a test either to earn a good grade or to avoid failing a course. Previous research has demonstrated different effects on cognition and behavior by motivation to earn rewards versus motivation to avoid punishments (Elliot 2008; Lang and Bradley 2010), suggesting that these states would also produce different effects on declarative learning. The goal of this study was to characterize the shared and specific effects of approach versus avoidance motivation on the encoding and use of declarative information, specifically information about spatial environments.

Previous research has demonstrated that motivation to learn enhances declarative memory. Specifically, reward incentives for successful encoding have been demonstrated to improve later memory retrieval (Adcock et al. 2006; Callan and Schweighofer 2008; Murayama and Kuhbandner 2011). However, extant human studies have only investigated approach motivation; to date, no parallel study has investigated the influence of avoidance

\footnotetext{
${ }^{6}$ Corresponding author.
}

E-mail alison.adcock@duke.edu.

Article is online at http://www.learnmem.org/cgi/doi/10.1101//m.023549.111. motivation — the opportunity to avoid punishments—on declarative learning. Approach and avoidance motivation have reliably been associated with distinct behaviors and neurophysiological states (Elliot 2008; Lang and Bradley 2010). Approach motivation is associated with behavioral activation, exploration, and positive affect (Ikemoto and Panksepp 1999), whereas avoidance motivation is associated with behavioral inhibition, stress, and anxiety (Davis and Whalen 2001; Lang and Bradley 2010). Given that these states differentially affect how individuals experience and interact with their environments, they should also differentially influence how individuals learn about their environments.

A paradigm widely used to investigate the encoding and subsequent retrieval of features of an environment, the Morris water task, also offers important advantages for human study. In this spatial learning paradigm, rodents are placed in a pool and must use stored spatial representations of the environment to navigate to a hidden platform. Rodent studies have demonstrated that performance on this task is sensitive to lesions of the hippocampus (Morris et al. 1982; Sutherland et al. 1982), a brain region known to support spatial and declarative learning (Eichenbaum 2004; Nadel and Hardt 2011). Like rodents, patients with hippocampal lesions or deficits show severe performance deficits on virtual reality versions of this task (Astur et al. 2002; Brandt et al. 2005). Thus, the virtual reality water task paradigm offers a valuable tool for connecting the human and rodent memory literatures (Hamilton et al. 2009), albeit with some debate regarding 
functional equivalence due to differences in stress elicited across species (de Quervain et al. 1998). Furthermore, because the experimental design of the water task integrates immediate goal pursuit (e.g., finding platforms) with declarative memory, it is well suited for manipulating reinforcement and goal-orientation without significantly modifying memoranda or experimental design. Together, these features make the human water task a useful tool for investigating the influence of approach and avoidance motivation on declarative learning.

In order to directly contrast effects of motivation by positive versus negative reinforcement on declarative learning, the current study modified the Morris water task to include two platforms, one good and one bad, for each incentive condition. For the approach condition, reaching the good platform earned a reward, and touching the bad one ended the trial. For the avoidance condition, contacts with the bad platform resulted in mild shock and reaching the good platform ended the trial. Given the spatial nature of this task, we were then able to assess the influence of motivation on learning and memory across multiple measures, including accuracy, learning rates, and precision. Additionally, we recorded participants' skin conductance levels (SCLs) during navigation in order to explicitly investigate relationships between arousal and motivated learning. Previous research has associated physiological arousal with both motivation (Low et al. 2008) and learning (Roozendaal et al. 2008). However, the relationships among arousal, motivational orientation, and declarative memory have not been explicated. By investigating the influence of both rewards and punishments on water task performance as well as concurrent SCLs, the present study tested the hypotheses that approach and avoidance motivation have differential influences on declarative memory, and that physiological arousal is an important determinant of how reinforcement affects motivated learning.

During each trial of the navigation task, participants were placed in a new start location in a virtual environment containing a pool with two hidden platforms: one correct and one incorrect (Supplemental Fig. 1). Participants navigated using a first-person perspective to learn the platform locations, in order to reach the correct platform while avoiding the incorrect platform. Trial-bytrial feedback guided learning. Each participant received five trials in four unique environments for a motivated and a nonmotivated condition, resulting in 20 trials per condition. In the motivated condition, the approach group of participants was rewarded with a $\$ 5$ monetary bonus each time they reached the correct platform, while the avoidance group was punished with a mild electrical shock each time they either touched the incorrect platform or failed to find the correct platform within $60 \mathrm{sec}$. We then measured memory for correct platform locations, path lengths to correct platform locations, learning rates, and spatial proximity to correct and incorrect platform locations for both motivated and nonmotivated conditions, in each of the approach and avoidance motivation groups.

Results demonstrated that both monetary bonuses for correct performance $\left(t_{(19)}=22.342, P<0.001\right)$ and electrical shocks for incorrect performance $\left(t_{(18)}=26.369, P<0.001\right)$ increased self-reports of motivation to perform, without any significant differences between groups $\left(t_{(37)}=-1.211, P=0.23\right)$. Behavioral performance was then compared between motivated and nonmotivated conditions across approach and avoidance motivation groups. No effects of participant gender on spatial memory, motivation, or their interactions $(P>0.15)$ were evident; however, sample sizes were relatively small to detect interactions between sex, motivation, and memory. Despite equivalent motivation, rewards promoted better spatial learning and memory than punishments. Specifically, participants found more correct platform locations under approach compared to avoidance motivation $\left(\right.$ group $\times$ condition interaction: $\left.\mathrm{F}_{(1,37)}=5.37, P=0.02\right)$ (Fig. 1A) Participants also showed a trend toward faster learning rates under approach compared to avoidance motivation, such that linear decreases in trial latencies were steeper during the pursuit of rewards (group $\times$ condition interaction: $\mathrm{F}_{(1,37)}=23.65, P=0.06$ ).

Further, participants demonstrated greater spatial precision in memory under approach compared to avoidance motivation. On correct trials, path lengths were shorter under approach compared to avoidance motivation $\left(F_{(1,37)}=4.64, P=0.04\right)$ (Fig. 1B). Additionally, during probe trials, in which participants were navigating in environments that did not contain any platforms, navigation in the proximity of correct and incorrect platforms was compared across groups. For this analysis, proximity was defined as the percentage of time spent navigating in the quadrant of the virtual environment containing each platform. This analysis revealed that approach-motivated participants spent relatively more time in the proximity of correct platform locations ([correct platform] $/$ [correct + incorrect] $)$ than avoidance motivated participants (group $\times$ condition interaction: $F_{(1,37)}=8.81, P=0.005$ ) (Fig. 1C). Critically, this relative difference across groups was driven by the avoidance group spending more time in the proximity of incorrect platform locations compared to the approach

A

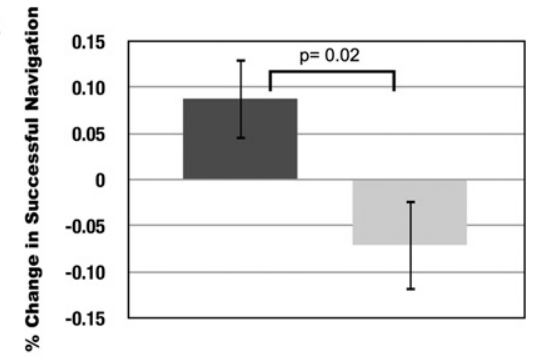

B
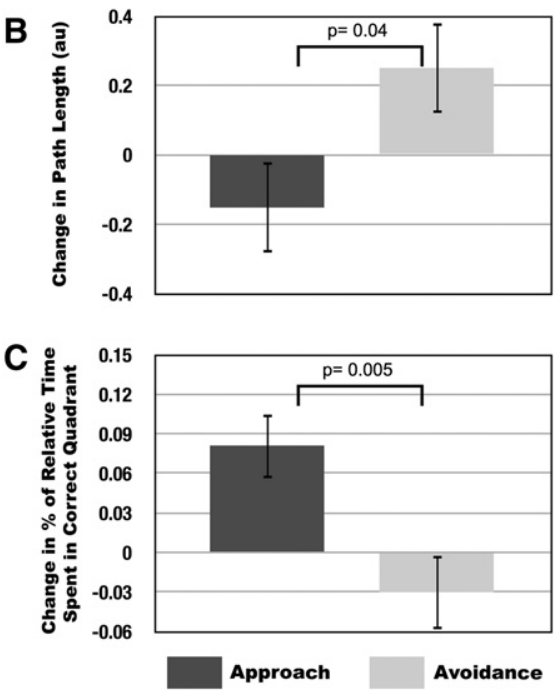

Figure 1. Spatial navigation performance across approach and avoidance groups: Mean difference scores are shown between motivated and nonmotivated conditions (error bars represent \pm SEM) in $(A)$ the mean percentage of trials resulting in navigation to correct platform locations, $(B)$ the mean path lengths (in arbitrary units) during navigation to correct platforms, and $(C)$ the percentage of time spent in the quadrant containing the correct platform, relative to the total time in quadrants containing the correct and incorrect platforms, during probe trials when no platforms were present in the environment. $P$-values reflect group by condition interactions. Note for panels $A$ and $B$, data from the first trial were not included as participants did not have the opportunity to learn. 
group $\left(F_{(1,37)}=5.58, P=0.02\right)$; there were no significant group differences in time spent in the proximity of correct platform locations $\left(F_{(1,37)}=2.597, P=0.12\right)$. The probe trial data argue against the explanation that avoidance-motivated participants might have neglected the correct platform locations due to a reinforcement bias toward avoiding the incorrect platform, and suggest instead that punishment resulted in a general deficit in learning.

Analysis of physiological data revealed that SCLs were higher in both motivated conditions compared to control conditions (main effect of condition: $F_{(1,34)}=11.21, P=0.002$ ) with no difference between groups (group $\times$ condition interaction: $F_{(1,34)}=$ $0.28, P=0.60$ ) (Fig. 3A, below). However, both within and across subjects, relationships between SCLs and memory were selective. For these analyses, memory was quantified as successful navigation to correct platform locations. Within subjects, SCLs were greater on incorrect than correct trials under approach but not avoidance motivation (group $\times$ condition interaction: $F_{(2,30)}=$ 5.712, $t_{(30)}=2.39, P=0.02$ ) (Fig. $2 \mathrm{~A}$ ). Across subjects, motivated SCL changes (motivated SCL-nonmotivated SCL) were inversely correlated with performance under approach $\left(r_{(16)}=-0.55, P=\right.$ $0.02)$ but not avoidance motivation $\left(r_{(16)}=0.04, P=0.86\right)$ (Fig. 2B), meaning that motivated-SCL responses were a better predictor of performance for approach-motivated compared to avoidance-motivated participants.

Thus, we demonstrated that low physiological arousal predicted memory success, but only under approach motivation. To confirm these findings, we compared motivated SCL changes (motivated SCL-nonmotivated SCL) between approach participants
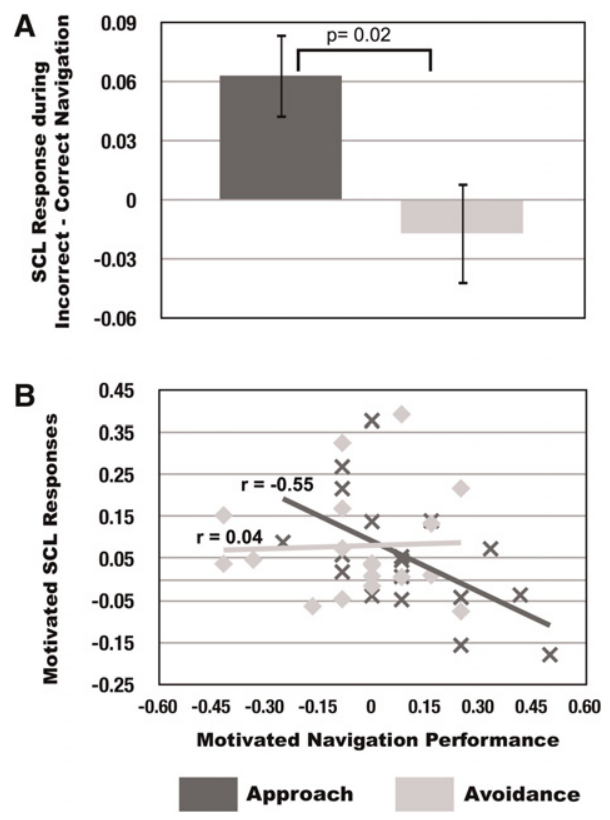

Figure 2. Relationships between spatial navigation performance (percent change in successful navigation) and physiological arousal responses (log-transformed $\mathrm{SCL}$ ) across approach and avoidance motivation groups. (A) Mean difference scores (error bars represent \pm SEM) for SCL responses during incorrect-correct platform locations were greater under approach compared to avoidance motivation (approach [incorrect SCL-correct SCL] > avoidance [incorrect SCL-correct SCL]). (B) Significant negative correlations were present between motivated SCL changes (SCL motivated trials-SCL nonmotivated trials) and spatial navigation performance (motivated correct platform navigation-nonmotivated correct platform navigation) under approach, but not avoidance, motivation.
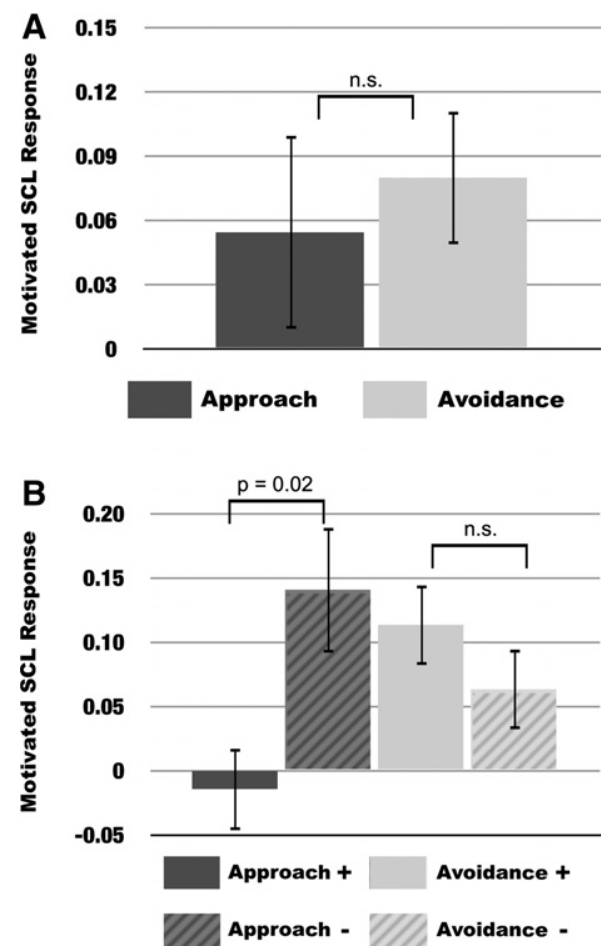

Figure 3. Motivated arousal responses across approach and avoidance motivation groups during navigation on standard trials. $(A)$ Mean differences (error bars represent \pm SEM) in motivated arousal changes (SCL motivated trials-SCL nonmotivated trials) across participants in the approach and avoidance motivation group. (B) Mean differences (error bars represent \pm SEM) of participants in the approach and avoidance motivation groups who showed performance benefits, defined as individuals whose navigation performance was greater in the motivated compared to the nonmotivated condition (approach,$+ n=10$; avoidance $+, n=6)$ as well as those who showed either performance deficits or no difference (approach - $n=8$; avoidance,$- n=12$ ).

who showed reward-motivated performance benefits (APP+) and those who did not (APP-). APP + participants were defined as individuals who found more correct platform locations in the approach motivation condition compared to the nonmotivated condition, whereas APP - participants were defined as individuals who showed any deficit, or no change, in the approach motivation condition. This analysis revealed that APP + participants had significantly lower motivated SCL changes than APP - participants $\left(t_{(16)}=-2.761, P=0.014\right)$ (Fig. 3B). A similar performance split in avoidance-motivated participants did not reveal any significant differences $\left(t_{(16)}=0.770, P=0.452\right)$ (Fig. 3B). There was also no difference in motivated SCL changes between APP - and avoidance motivated participants $\left(t_{(24)}=1.082, P=0.29\right)$, suggesting that these two groups had similar arousal profiles during learning.

The present study provides a novel characterization of how motivation to avoid punishments influences declarative learning in humans, and further provides evidence for differential effects of approach and avoidance motivation on spatial learning. Overall, the pursuit of monetary rewards resulted in better memory than the avoidance of electrical stimulation. Further, the current study demonstrated a critical detrimental role for physiological arousal in motivated learning. Specifically, high arousal was associated with poor performance under reward motivation. This relationship was not evident for avoidance-motivated participants, who, as a group, showed worse performance. Finally, 
the study demonstrated that low-performing approach-motivated participants had arousal profiles similar to avoidance-motivated participants.

This pattern of facilitation by approach motivation and impairment by avoidance motivation during declarative learning differs from investigations of motivated learning in other domains. For simple associative processes, such as stimulus-response learning (Delgado et al. 2008; Robinson et al. 2010; Prevost et al. 2011) and Pavlovian conditioning (Gottfried et al. 2002; Seymour et al. 2007; Pessiglione et al. 2008), earning rewards and avoiding punishments can both facilitate learning. However, Pavlovian and stimulus-response learning fundamentally differ from declarative memory, in which environmental features are encoded into long-term mnemonic representations. Further, these separable kinds of learning are mediated by parallel neural systems (Iaria et al. 2003; Poldrack and Packard 2003). Specifically, declarative learning is supported by the hippocampus (Eichenbaum 2004), as both humans and rodents with hippocampus lesions show severe deficits in declarative memory tasks, including spatial learning (Morris et al. 1982; Sutherland et al. 1982; Astur et al. 2002). Conversely, patients with hippocampal lesions do not show significant deficits in stimulus-response learning or simple Pavlovian learning (Squire 1992). Together these findings suggest that the influence of approach and avoidance motivation may be mediated by the specific neural substrate mediating learning, such that positive and negative reinforcements have divergent influences on hippocampus-dependent, declarative memory. However, future studies will need to directly compare positive and negative reinforcement on other forms of declarative memory, such as item memory or paired-associate learning.

The current study also identified a unique role for physiological arousal in mediating motivated learning. Separate lines of research have previously highlighted the role of arousal in learning and memory, and in motivated behavior. Regarding spatial memory, rodent studies have demonstrated that increases in physiological arousal, induced by either physiological or psychosocial stress, disrupt hippocampus-dependent declarative memory and promote stimulus-response learning in water maze paradigms (Kim et al. 2001, 2005; Roozendaal et al. 2003). Both approach and avoidance motivations have been associated with increases in physiological arousal (Low et al. 2008; Mobbs et al. 2009); however, these studies did not investigate how changes in arousal related to goal-directed learning. Consistent with these prior findings, here both shocks and rewards elicited equivalent SCLs; differences across motivation conditions only emerged when relating SCL to motivated memory performance. The current study demonstrates a novel aspect of approach motivation: If an individual responds to an incentive with high arousal, as typifies avoidance motivation, performance will not benefit and may in fact suffer. These findings suggest that the subgroup of approach-motivated individuals who showed performance deficits may also be in a state typically elicited by avoidance motivation. Outside of the domain of learning, reward motivation has been shown to elicit performance deficits in some individuals. Previous investigators have related this behavior to "choking under pressure," in which performance drops when reward incentives evoke stress responses and anxiety (Ariely et al. 2009; Mobbs et al. 2009). One memory study has demonstrated anxietyinduced performance deficits in some subjects during rewardmotivated declarative learning (Callan and Schweighofer 2008); however, that study relied on self-report of anxiety given after the experimental task. The current study's findings advance the literature by directly relating dynamic shifts in SCLs to memory performance during reward-motivated learning both across and within subjects. The results point to physiological arousal as a potentially important inhibitor of motivated learning, with similar learning deficits in highly aroused appetitive states and in avoidance.

The relationship between physiological arousal and motivated memory was specific to reward motivation. Despite equivalent overall arousal in the two groups, neither within- nor across-subject analysis revealed any significant relationships between spatial navigation accuracy and physiological arousal under avoidance motivation. This pattern of findings may reflect greater heterogeneity in the motivated learning states evoked by approach incentives compared to avoidance incentives. That is, avoidance incentives may consistently evoke a state associated with physiological arousal, anxiety, and impaired learning, such that variability in these markers during avoidance motivation is not predictive of learning. Conversely, approach incentives evoke states of anxiety only in some behavioral contexts, such that physiological arousal indexes variation in reward-induced stress, and thus also reliably indexes variation in reward-induced learning impairment. Thus, in line with the stress and spatial navigation literature, we propose a model of motivated learning in which arousal dictates the efficacy of hippocampus-dependent spatial learning. However, our findings suggest that whereas avoidance motivation reliably evokes states of stress, approach motivation only evokes these states in subgroups of participants. Future studies will need to investigate the relationships between positive and negative incentives, self-reported anxiety, and physiological markers such as SCL.

Although our study provided novel evidence regarding the influence of approach and avoidance motivation on declarative memory, one aspect of our current experimental design limits the interpretation of our findings. This current study used electrical shocks instead of monetary losses to induce avoidance motivation, as the threat of shock more reliably activates circuitry implicated in avoidance motivation (Delgado et al. 2008, 2011); however, this resulted in qualitatively different reinforcers, namely, electrical stimulation versus monetary bonuses. Although these reinforcers induced similar self-reports of motivation and SCLs, they fundamentally differ in that electrical stimulations are primary reinforcers whereas monetary rewards are secondary reinforcers. Although some studies have provided evidence that primary and secondary reinforcers are processed very similarly in the brain (O'Doherty and Valentin 2009; Kim et al. 2011), other studies have noted significant differences (Beck et al. 2010). Future studies using reinforcers within a similar qualitative domain, such as aversive and appetitive odors or juices, should test the prediction that, irrespective of valence, if a motivational state is accompanied by high arousal, hippocampal learning will be impaired, not enhanced.

In conclusion, we provide a novel characterization of how avoiding punishments influences declarative learning and demonstrate that approach and avoidance motivation have differential influences on declarative learning and memory. Further, we demonstrate that individual interpretation of incentives is an important mediator of this effect. In general, approach motivation enhanced but avoidance motivation interfered with the successful encoding and use of environmental representations. The benefits of approach motivation were not seen in those individuals who became physiologically aroused during the pursuit of rewards. Together these findings support a model in which how one interprets and responds to an incentive, rather than its objective value, is what dictates the efficacy of learning. Future studies investigating the psychological and neural factors that determine the efficacy of learning incentives will contribute to better understanding of how to motivate successful declarative learning for a given individual. Individual differences notwithstanding, however, these data suggest that for declarative learning, specifically spatial learning, reward is a better incentive than threat. 


\section{Materials and Methods}

Forty-two healthy, right-handed participants received $\$ 10 / \mathrm{h}$ plus any monetary bonuses for study participation. Participants were randomly assigned to either approach or avoidance motivation groups. Data from three participants were excluded for computer malfunctions or poor baseline performance (less than or equal to chance), resulting in 39 participants (median age $=$ 22 , age range $=19-28), 19$ participants in the approach group (seven female) and 20 participants in the avoidance group (10 female).

Participants performed a spatial-memory task in both motivated and nonmotivated conditions, with motivational reinforcement (i.e., approach or avoidance) as a between-subjects factor. In motivated conditions, approach participants were given $\$ 5$ monetary bonuses for correct performance and avoidance participants were given electrical shocks for incorrect performance. Electrical shocks, as opposed to monetary loses, were used because previous research has demonstrated that approach motivation networks are similarly engaged when individuals prepare to gain monetary rewards and avoid monetary losses (Carter et al. 2009) and electrical shocks more reliably elicit activation in avoidance motivation neural circuitry (Delgado et al. 2008, 2011). Participants completed four blocks (four standard trials and one probe trial, 10 sec interstimulus intervals [ISIs]) of spatial navigation under motivated and nonmotivated conditions. Standard trials ended when the participants reached either the correct or incorrect platform or $60 \mathrm{sec}$ passed, at which point the correct platform became visible and the trial was considered incorrect. At the end of each trial, participants viewed visual feedback regarding their trial performance: text reading "correct," "incorrect," or "correct platform visible." Participants entered the pool at a different location on each trial and locations stayed constant in a given environment. During probe trials, participants navigated for $60 \mathrm{sec}$ in an environment that did not contain any platforms, to allow for the measurement of spatial navigation performance over a fixed-time period.

An MP-150 and MP-100 BIOPAC system (BIOPAC Systems) was used both for the administration of shocks and the recording of SCL responses, respectively (see Dunsmoor et al. 2009 for further details). Shocks were calibrated to a level that was "highly irritating but not painful" using an ascending staircase procedure. SCLs were sampled and recorded at $200 \mathrm{~Hz}$ from the second and third digits of the left hand from trial onset to platform discovery, excluding time epochs containing reinforcement. SCLs were normalized by log-transformation. Three participants' SCL data were not collected due to technical issues. Following completion of the behavioral paradigm, participants completed study-specific, selfreport questionnaires to quantify individuals' motivation to perform during the incentivized condition. Specifically, participants were asked "Were you more motivated to perform during the shocked/reward session?" and indicated their responses on a fivepoint scale ranging from "not at all" to "yes."

Statistical significance for spatial memory performance and SCLs was tested using general linear models with condition (motivated, nonmotivated) as a within-subjects factor and group (approach, avoidance) as a between-subjects factor. Accuracy and precision data from the first trial were excluded from statistical analysis because performance was at chance. Learning rate was quantified as the $\beta$-weight from a regression between platform latency and trial number, with incorrect trials coded as 60-sec latencies. Probe trials were analyzed by calculating the relative time participants spent in the quadrant of correct compared to incorrect platforms. Relationships between arousal, motivation, and behavioral performance were analyzed using three different techniques. First, SCL measures were entered into a general linear model with trial performance (correct or incorrect) as a within-subjects factor and group (approach or avoid) as a between-subjects factor. Second, differences in SCL measures across condition (motivated-nonmotivated SCL) were regressed against motivated-memory performance for approach and avoidance groups, separately. Finally, differences in SCL measures across condition (motivated-nonmotivated SCL) were compared across low-performing and high-performing groups using twosample $t$-tests for approach and avoidance groups, separately. Results from all statistical analyses were considered significant at a level of $P<0.05$.

\section{Acknowledgments}

We thank Katherine MacDuffie and Elizabeth Johnson for helpful discussions and comments. This study was supported by National Institutes of Drug Addiction grant RO1DA027802. R.A.A. was supported in part by the Alfred P. Sloan Foundation.

\section{References}

Adcock RA, Thangavel A, Whitfield-Gabrieli S, Knutson B, Gabrieli JD. 2006. Reward-motivated learning: Mesolimbic activation precedes memory formation. Neuron 50: 507-517.

Ariely D, Gneezy U, Loewenstein G, Mazar N. 2009. Large stakes and big mistakes. Rev Econ Stud 76: 451-469.

Astur RS, Taylor LB, Mamelak AN, Philpott L, Sutherland RJ. 2002. Humans with hippocampus damage display severe spatial memory impairments in a virtual Morris water task. Behav Brain Res 132: 77-84.

Beck SM, Locke HS, Savine AC, Jimura K, Braver TS. 2010. Primary and secondary rewards differentially modulate neural activity dynamics during working memory. Plos One 5: e9251. doi: 10.1371/ journal.pone.0009251.

Brandt T, Schautzer F, Hamilton DA, Bruning R, Markowitsch HJ, Kalla R, Darlington C, Smith P, Strupp M. 2005. Vestibular loss causes hippocampal atrophy and impaired spatial memory in humans. Brain 128: $2732-2741$.

Callan DE, Schweighofer N. 2008. Positive and negative modulation of word learning by reward anticipation. Hum Brain Mapp 29: 237-249.

Carter RM, Macinnes JJ, Huettel SA, Adcock RA. 2009. Activation in the VTA and nucleus accumbens increases in anticipation of both gains and losses. Front Behav Neurosci 3: 21.

Davis M, Whalen PJ. 2001. The amygdala: Vigilance and emotion. Mol Psychiatry 6: $13-34$

Delgado MR, Li J, Schiller D, Phelps EA. 2008. The role of the striatum in aversive learning and aversive prediction errors. Philos Trans $R$ Soc Lond B Biol Sci 363: 3787-3800.

Delgado MR, Jou RL, Phelps EA. 2011. Neural systems underlying aversive conditioning in humans with primary and secondary reinforcers. Front Neurosci 5: 71.

de Quervain DJ, Roozendaal B, McGaugh JL. 1998. Stress and glucocorticoids impair retrieval of long-term spatial memory. Nature 394: $787-790$.

Dunsmoor JE, Mitroff SR, LaBar KS. 2009. Generalization of conditioned fear along a dimension of increasing fear intensity. Learn Mem 16: $460-469$.

Eichenbaum H. 2004. Hippocampus: Cognitive processes and neural representations that underlie declarative memory. Neuron 44: $109-120$.

Elliot AJ. 2008. Approach and avoidance motivation. In Handbook of approach and avoidance motivation (ed. AJ Elliot), pp. 3-16. Psychology Press, New York.

Gottfried JA, O'Doherty J, Dolan RJ. 2002. Appetitive and aversive olfactory learning in humans studied using event-related functional magnetic resonance imaging. I Neurosci 22: 10829-10837.

Hamilton DA, Johnson TE, Redhead ES, Verney SP. 2009. Control of rodent and human spatial navigation by room and apparatus cues. Behav Processes 81: 154-169.

Iaria G, Petrides M, Dagher A, Pike B, Bohbot VD. 2003. Cognitive strategies dependent on the hippocampus and caudate nucleus in human navigation: Variability and change with practice. J Neurosci 23: 5945-5952.

Ikemoto S, Panksepp J. 1999. The role of nucleus accumbens dopamine in motivated behavior: A unifying interpretation with special reference to reward-seeking. Brain Res Brain Res Rev 31: 6-41.

Kim JJ, Lee HJ, Han JS, Packard MG. 2001. Amygdala is critical for stress-induced modulation of hippocampal long-term potentiation and learning. J Neurosci 21: 5222-5228.

Kim JJ, Koo JW, Lee HJ, Han JS. 2005. Amygdalar inactivation blocks stress-induced impairments in hippocampal long-term potentiation and spatial memory. J Neurosci 25: 1532-1539.

Kim H, Shimojo S, O'Doherty JP. 2011. Overlapping responses for the expectation of juice and money rewards in human ventromedial prefrontal cortex. Cereb Cortex 21: 769-776.

Lang PJ, Bradley MM. 2010. Emotion and the motivational brain. Biol Psychol 84: $437-450$. 
Low A, Lang PJ, Smith JC, Bradley MM. 2008. Both predator and prey: Emotional arousal in threat and reward. Psychol Sci 19: $865-873$.

Mobbs D, Hassabis D, Seymour B, Marchant JL, Weiskopf N, Dolan RJ, Frith CD. 2009. Choking on the money: Reward-based performance decrements are associated with midbrain activity. Psychol Sci 20: 955-962.

Morris RG, Garrud P, Rawlins JN, O'Keefe J. 1982. Place navigation impaired in rats with hippocampal lesions. Nature 297: 681-683.

Murayama K, Kuhbandner C. 2011. Money enhances memory consolidation-but only for boring material. Cognition 119: 120-124.

Nadel L, Hardt O. 2011. Update on memory systems and processes. Neuropsychopharmacology 36: 251-273.

O'Doherty JP, Valentin VV. 2009. Overlapping prediction errors in dorsal striatum during instrumental learning with juice and money reward in the human brain. J Neurophysiol 102: 3384-3391.

Pessiglione M, Petrovic P, Daunizeau J, Palminteri S, Dolan RJ, Frith CD. 2008. Subliminal instrumental conditioning demonstrated in the human brain. Neuron 59: 561-567.

Poldrack RA, Packard MG. 2003. Competition among multiple memory systems: Converging evidence from animal and human brain studies. Neuropsychologia 41: 245-251.

Prevost C, McCabe JA, Jessup RK, Bossaerts P, O’Doherty JP. 2011. Differentiable contributions of human amygdalar subregions in the computations underlying reward and avoidance learning. Eur I Neurosci 34: $134-145$.

Robinson OJ, Frank MJ, Sahakian BJ, Cools R. 2010. Dissociable responses to punishment in distinct striatal regions during reversal learning. Neuroimage 51: 1459-1467.

Roozendaal B, Griffith QK, Buranday J, De Quervain DJ, McGaugh JL. 2003. The hippocampus mediates glucocorticoid-induced impairment of spatial memory retrieval: Dependence on the basolateral amygdala. Proc Natl Acad Sci 100: $1328-1333$.

Roozendaal B, Barsegyan A, Lee S. 2008. Adrenal stress hormones, amygdala activation, and memory for emotionally arousing experiences. Prog Brain Res 167: 79-97.

Seymour B, Daw N, Dayan P, Singer T, Dolan R. 2007. Differential encoding of losses and gains in the human striatum. INeurosci 27: 4826-4831.

Shohamy D, Adcock RA. 2010. Dopamine and adaptive memory. Trends Cogn Sci 14: 464-472.

Squire LR. 1992. Memory and the hippocampus: A synthesis from findings with rats, monkeys, and humans. Psychol Rev 99: 195-231.

Sutherland RJ, Kolb B, Whishaw IQ. 1982. Spatial mapping: Definitive disruption by hippocampal or medial frontal cortical damage in the rat. Neurosci Lett 31: 271-276.

Received July 15, 2011; accepted in revised form September 23, 2011. 


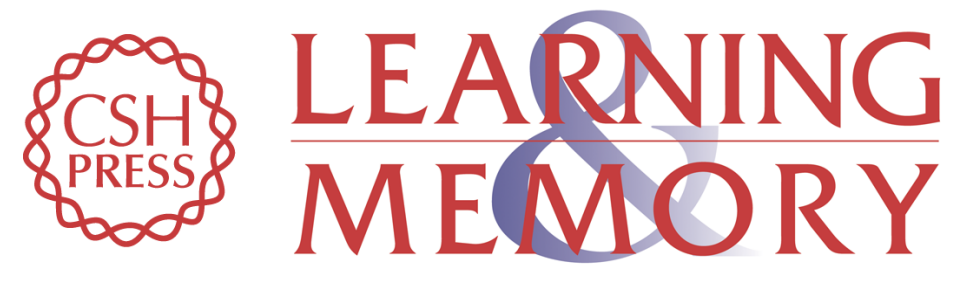

\section{Is all motivation good for learning? Dissociable influences of approach and avoidance motivation in declarative memory}

Vishnu P. Murty, Kevin S. LaBar, Derek A. Hamilton, et al.

Learn. Mem. 2011, 18:

Access the most recent version at doi:10.1101//m.023549.111

Supplemental
Material http://learnmem.cshlp.org/content/suppl/2011/10/21/18.11.712.DC1

References This article cites 37 articles, 7 of which can be accessed free at: http://learnmem.cshlp.org/content/18/11/712.full.html\#ref-list-1

License

Email Alerting Receive free email alerts when new articles cite this article - sign up in the box at the Service top right corner of the article or click here. 